Energietechnologien der Zukunft 
Martin Wietschel . Sandra Ullrich •

Peter Markewitz • Friedrich Schulte .

Fabio Genoese

Herausgeber

\section{Energietechnologien der Zukunft}

Erzeugung, Speicherung, Effizienz und Netze

Springer Vieweg 


\section{Herausgeber}

Martin Wietschel

Fraunhofer-Institut für System- und

Innovationsforschung ISI

Karlsruhe, Deutschland

Sandra Ullrich

Fraunhofer-Institut für System- und

Innovationsforschung ISI

Karlsruhe, Deutschland

\author{
Peter Markewitz \\ Forschungszentrum Jülich $\mathrm{GmbH}$ \\ Jülich, Deutschland \\ Friedrich Schulte \\ RWE AG \\ Essen, Deutschland \\ Fabio Genoese \\ Centre for European Policy Studies (CEPS) \\ Brüssel, Belgien
}

ISBN 978-3-658-07128-8

ISBN 978-3-658-07129-5 (eBook)

DOI 10.1007/978-3-658-07129-5

Die Deutsche Nationalbibliothek verzeichnet diese Publikation in der Deutschen Nationalbibliografie; detaillierte bibliografische Daten sind im Internet über http://dnb.d-nb.de abrufbar.

Springer Vieweg

(C) Springer Fachmedien Wiesbaden 2015

Das Werk einschließlich aller seiner Teile ist urheberrechtlich geschützt. Jede Verwertung, die nicht ausdrücklich vom Urheberrechtsgesetz zugelassen ist, bedarf der vorherigen Zustimmung des Verlags. Das gilt insbesondere für Vervielfältigungen, Bearbeitungen, Übersetzungen, Mikroverfilmungen und die Einspeicherung und Verarbeitung in elektronischen Systemen.

Die Wiedergabe von Gebrauchsnamen, Handelsnamen, Warenbezeichnungen usw. in diesem Werk berechtigt auch ohne besondere Kennzeichnung nicht zu der Annahme, dass solche Namen im Sinne der Warenzeichen- und Markenschutz-Gesetzgebung als frei zu betrachten wären und daher von jedermann benutzt werden dürften.

Der Verlag, die Autoren und die Herausgeber gehen davon aus, dass die Angaben und Informationen in diesem Werk zum Zeitpunkt der Veröffentlichung vollständig und korrekt sind. Weder der Verlag noch die Autoren oder die Herausgeber übernehmen, ausdrücklich oder implizit, Gewähr für den Inhalt des Werkes, etwaige Fehler oder Äußerungen.

Lektorat: Dr. Daniel Fröhlich

Gedruckt auf säurefreiem und chlorfrei gebleichtem Papier.

Springer Fachmedien Wiesbaden GmbH ist Teil der Fachverlagsgruppe Springer Science+Business Media (www.springer.com) 


\section{Vorwort}

Energie ist ein wesentlicher strategischer Faktor für Wirtschaft und Gesellschaft. Die Herausforderungen des Klimaschutzes, die Verknappung der fossilen Energieträger und die Abkehr von der Kernenergie haben zu einem Transformationsprozess in der Energiewirtschaft geführt. Innovative Energietechnologien in Kombination mit dezentralen Strukturen von Erzeugung und Verteilung gewinnen im zukünftigen Energiesystem immer stärker an Relevanz. Der Marktanteil der dargebotsabhängigen erneuerbaren Energieträger nimmt stetig zu.

In diesem Buch wird zuerst das zukünftige Elektrizitätssystem in einem systemischen Ansatz auf Basis der erwarteten politischen, gesellschaftlichen und technologischen Entwicklungen skizziert. Darüber hinaus werden wichtige innovative Energietechnologien in einem künftigen deutschen und europäischen Elektrizitätssystem identifiziert. Daran anschließend werden für diese Technologien der heutige technische und ökonomische Entwicklungsstand sowie das Entwicklungspotenzial in den nächsten 10 bis 15 Jahren von verschiedenen Experten aus renommierten Forschungseinrichtungen dargelegt. Der künftige F\&E-Bedarf mit den wesentlichen Trends wird skizziert. Die Technologiefelder werden abschließend in Roadmaps übersichtlich anhand ihrer technischen und wirtschaftlichen Entwicklungsziele, ihres zeitlich verorteten F\&E-Bedarfes, ihrer Marktrelevanz sowie ihrer wichtigsten Treiber und Hemmnisse dargestellt. Aufgrund der aktuell sehr vielfältigen und schnellen Entwicklungen bei den Energietechnologien kann kein Anspruch auf absolute Vollständigkeit in den Darstellungen und Erläuterungen erhoben werden.

Dieses Buch soll zur Grundlageninformation für Wirtschaft, Politik und Wissenschaft dienen und helfen, die entscheidenden Diskussionen und Maßnahmen über langfristige Handlungs- und Entscheidungsspielräume voran zu treiben. 
Wesentliche Inhalte des Buches entstanden im Rahmen eines Forschungsprojektes im Jahr 2013 für die Forschungs- und Entwicklungsabteilung der RWE AG, die einer Veröffentlichung dankenswerter Weise zugestimmt hat. Die Verantwortung für die Inhalte liegt aber alleine bei den Autoren der Kapitel. Ein Vorläuferprojekt mit dem Titel Energietechnologien 2050 - Schwerpunkte für Forschung und Entwicklung wurde vom Bundesministerium für Wirtschaft und Technologie gefördert und hat Eingang in das 6. Energieforschungsprogramm der Bundesregierung gefunden.

Die Herausgeber 


\section{Über die Autoren}

\section{M .Sc. Wi.-Ing. Ali Aydemir}

Studium des Wirtschaftsingenieurwesens mit Schwerpunkt „Energie- und Verfahrenstechnik“ an der Universität Duisburg-Essen (Abschluss M. Sc. Wi.-Ing 2010). Von 2011 bis 2013 tätig für die Siemens AG als Vertriebsingenieur für den Verdichter-Service in Duisburg. Seit Februar 2013 wissenschaftlicher Mitarbeiter am Fraunhofer Institut für System- und Innovationsforschung im Competence Center Energietechnologien und Energiesysteme.

Internet: www.isi.fraunhofer.de

Kapitel 17: Raumlufttechnik und Klimakältesysteme

Kapitel 18: Wärmepumpen

\section{Dipl.-Wirtsch.ing. (FH) Tobias Bischkowski}

Tobias Bischkowski, Hauptreferent im Bereich Steuerung der Konzern Forschung \& Entwicklung (F\&E) der RWE AG, ist seit 2005 im RWE Konzern tätig. Er war bis zuletzt mitverantwortlich für die prozessuale Steuerung und Weiterentwicklung des F\&E-Projektportfolios. Zuvor war er bei RWE mit der Analyse und Bewertung von Energietechnologien und -szenarien betraut. 2012 erarbeitete er das Konzept der Technologie-Roadmaps als Planungsinstrument der F\&E bei RWE.

Bevor Herr Bischkowski im Jahr 2005 in der Internen Revision bei RWE anfing, war er zu Beginn seiner beruflichen Laufbahn vier Jahre als Unternehmensberater in den Bereichen Risk Advisory Services und Business Process Risk Consulting bei den Ernst \& Young und Arthur Andersen tätig.

Aktuell ist er bei RWE projektbezogen im Umfeld von Lean Management und Veränderungsprozessen tätig. Tobias Bischkowski ist Diplom-Wirtschaftsingenieur (FH) mit den Studienschwerpunkten im Controlling und Marketing.

Internet: www.rwe.com

Kapitel 1: Motivation, Zielsetzung und Technologieauswahl 


\section{Dipl.-Ing. Karl Ulf Birnbaum}

Studium Maschinenbau und Brennstoffingenieurwesen an der RWTH Aachen. Von 1979 bis 2013 wissenschaftlicher Mitarbeiter am Forschungszentrums Jülich, Institut für Energie- und Klimaforschung - Systemforschung und technologische Entwicklung (IEKSTE).

Schwerpunkte: Konventionelle Kraftwerkstechnik, Mikro-KWK (Haushalte, Kleinverbraucher), Wasserstofferzeugung und -nutzung, Energieversorgungsszenarien.

Internet: www.fz-juelich.de

Kapitel 2: Zukünftige Energiewelt - Szenarien und robuste Trends

Kapitel 16: Mikro-Kraftwärmekopplungsanlagen (Mikro-KWK)

\section{Dr. rer. nat. Klaus Hendrik Biß}

Abschluss des Physikstudiums an der Justus-Liebig Universität Gießen. Von 2010 bis 2012 Doktorand am Lehrstuhl Nukleare Entsorgung und Techniktransfer (NET). Promotion im Jahr 2014 an der RWTH Aachen. Seit 2012 wissenschaftlicher Mitarbeiter am Forschungszentrums Jülich, Institut für Energie- und Klimaforschung - Systemforschung und technologische Entwicklung (IEK-STE).

Schwerpunkte: Energiesystemmodellierung, Endenergiebedarfsanalyse, Energieszenarien.

Internet: www.fz-juelich.de

Kapitel 2: Zukünftige Energiewelt - Szenarien und robuste Trends

Kapitel 3: Kohlekraftwerke

Kapitel 4: Gaskraftwerke

Kapitel 5: $\mathrm{CO}_{2}$-Abscheidung

Kapitel 6: $\mathrm{CO}_{2}$-Nutzung

\section{Dipl.-Ing. Richard Bongartz}

Studium der Kernverfahrenstechnik an der Fachhochschule Aachen. Von 1975 bis 2014 wissenschaftlicher Mitarbeiter am Forschungszentrums Jülich, seit 2008 am Institut für Energie- und Klimaforschung - Systemforschung und technologische Entwicklung (IEKSTE).

Schwerpunkte: Anlagen der Verfahrenstechnik; technische Bewertung von Energietechnologien, Risikobewertung.

Internet: www.fz-juelich.de

Kapitel 3: Kohlekraftwerke

Kapitel 4: Gaskraftwerke

Kapitel 5: $\mathrm{CO}_{2}$-Abscheidung

Kapitel 6: $\mathrm{CO}_{2}$-Nutzung

Kapitel 16: Mikro-Kraftwärmekopplungsanlagen (Mikro-KWK) 


\section{Dr.-Ing. Reiner Buck}

Studium des Maschinenbaus mit Schwerpunkt Energietechnik an der Universität Stuttgart. Promotion im Jahr 2000 zum Thema „Massenstrom-Instabilitäten bei volumetrischen Receiver-Reaktoren“. Seit 1986 als wissenschaftlicher Mitarbeiter am Deutschen Zentrum für Luft- und Raumfahrt e. V. (DLR) tätig, zunächst am Institut für Technische Thermodynamik, seit 2011 am Institut für Solarforschung.

Seit 2011 Leiter der Abteilung „Punktfokussierende Systeme“ am DLR-Institut für Solarforschung. Schwerpunkte: Entwicklung von konzentrierenden Solarsystemen zur Stromerzeugung, Thermodynamik, System-Auslegung und -Modellierung, optische Konzentratoren. Mitarbeit und Leitung diverser nationaler und internationaler Projekte im Bereich konzentrierender Solarsysteme zur Stromerzeugung.

Internet: www.dlr.de

Kapitel 9: Solarthermische Kraftwerke

\section{Prof. Dr.-Ing. Martin Braun}

Studium der „Elektro- und Informationstechnik“ und ,technisch orientierte Betriebswirtschaftslehre“ an der Universität Stuttgart. In den Jahren 2005-2008 wissenschaftlicher Mitarbeiter am Institut für Solare Energieversorgungstechnik (ISET) in Kassel in der Gruppe „Elektrische Netze“. 2008 Promotion an der Universität Kassel über das Thema „Bereitstellung von Netzdienstleistungen durch dezentrale Erzeuger“. Ab 2009 Aufbau der Forschungsgruppe „Dezentrale Netzdienstleistungen“ am Fraunhofer-Institut für Windenergie und Energiesystemtechnik (IWES) in Kassel. 2010-2012 Juniorprofessor für „Smart Power Grids“ an der Universität Stuttgart. Seit 2012 Professor an der Universität Kassel, Fachgebietsleiter „Energiemanagement und Betrieb elektrischer Netze“ verbunden mit dem Kompetenzzentrum für Dezentrale Elektrische Energieversorgungstechnik (KDEE). Seit 2012 Abteilungsleiter „Betrieb Verteilungsnetze“ am Fraunhofer IWES. Forschungsschwerpunkte sind technisch-wirtschaftlich optimierte Verfahren für die Analyse, Auslegung, Regelung und Betriebsführung von Verteilungsnetzen sowie die Bereitstellung von Energie- und Netzdienstleistungen durch dezentrale Anlagen und Verfahren für Energie- und Netzmanagement in dezentralen Versorgungsstrukturen.

Internet: www.uni-kassel.de, www.iwes.fraunhofer.de

Kapitel 15: Elektrische Verteilungsnetze im Wandel

\section{Dipl.-Ing. Johannes Fleer}

Studium Umwelttechnik und Ressourcenmanagement an der Ruhr-Universität Bochum. Anschließend Tätigkeit als Planungsingenieur für Photovoltaiksysteme bei der Firma ecoKinetics Pty Ltd in Stapylton, Australien. Seit 2012 wissenschaftlicher Mitarbeiter am Forschungszentrums Jülich, Institut für Energie- und Klimaforschung - Systemforschung und technologische Entwicklung (IEK-STE). 
Schwerpunkte: Techno-ökonomische Analyse und Bewertung von Energiespeichern, Analyse des Einsatzes netzgekoppelter Batteriespeichersysteme zur Bereitstellung von Regelleistung.

Internet: www.fz-juelich.de

Kapitel 10: Elektrochemische Speicher

\section{Dr. Tobias Fleiter}

Tobias Fleiter studierte Wirtschaftsingenieurwesen „Energie- und Umweltmanagement“ an der Universität Flensburg mit Studienaufenthalt in Schweden. Seit 2007 ist Tobias Fleiter am Fraunhofer Institut für System- und Innovationsforschung im Competence Center Energietechnologien und Energiesysteme tätig. Währenddessen hat er einen Aufenthalt bei der Europäischen Kommission in der Generaldirektion für Energie- und Transport absolviert und war als Gastwissenschaftler an der Universität Bordeaux IV sowie am IPM der Chinese Academy of Science in Peking. Seine Doktorarbeit hat er an der Universität Utrecht zum Thema „Die Adoption von Energieeffizienztechnologien durch Unternehmen“ im Jahr 2012 abgeschlossen. Seit 2013 ist Tobias Fleiter Leiter des Geschäftsfeldes Energienachfrageanalysen und -projektionen. Seine Forschungsschwerpunkte sind die Modellierung der Energienachfrage sowie Energieeffizienz in der Industrie. Dies beinhaltet sowohl techno-ökonomische Bewertungen von Energieeinsparpotenzialen als auch Wirkungsanalysen von Instrumenten zur Steigerung der Energieeffizienz.

Internet: www.isi.fraunhofer.de

Kapitel 19: Stromeffizienz in den Sektoren Industrie, GHD und Haushalte

\section{Dr. Nele Friedrichsen}

Studium Wirtschaftsingenieurswesen mit Schwerpunkt Energie- und Umweltmanagement an der Universität Flensburg. Promotion in VWL an der Jacobs University Bremen. Von 2010 bis 2012 wissenschaftliche Mitarbeiterin am Bremer Energie Institut. Seit 2013 wissenschaftliche Mitarbeiterin am Fraunhofer Institut für System- und Innovationsforschung. Bis Juni 2014 im Competence Center Energietechnologien und Energiesysteme, danach im Competence Center Energiepolitik und Energiemärkte. Ihre Interessenschwerpunkte sind institutionelle Rahmenbedingungen und Governance insbesondere hinsichtlich der Transition zu einem nachhaltigen Energiesystem. Am Fraunhofer ISI beschäftigt sie sich derzeit mit der Ausgestaltung und Bewertung des EU-Emissionsrechtehandels, dem Netzentgeltsystem im Kontext der Energiewende und den damit verbundenen Herausforderungen sowie der Wettbewerbsfähigkeit der deutschen energieintensiven Industrie.

Internet: www.isi.fraunhofer.de

Kapitel 20: Verbrauchssteuerung

\section{Dr. Fabio Genoese}

Dr. Fabio Genoese ist CEPS Fellow am Centre for European Policy Studies in Brüssel und Dozent am Institut d'études politiques (Sciences Po) in Paris. Seine Forschungs- 
schwerpunkte sind der EU-Binnenmarkt für Strom und Gas, eine EU-weite Betrachtung der Energiewende sowie die Bedeutung neuer Energietechnologien zum Voranbringen der Energiewende. Von 2008 bis 2013 arbeitete er am Fraunhofer ISI in Karlsruhe, wo er für Forschungs- und Beratungsprojekte für öffentliche Einrichtungen und Unternehmen in der Energiebranche leitete. Im Jahr 2012 war er als Gastwissenschaftler bei Fondazione Eni Enrico Mattei (FEEM) in Mailand. Er ist promovierter Wirtschaftswissenschaftler.

Internet: www.ceps.eu

Kapitel 11: Druckluftspeicher

Kapitel 12: Power-to-Gas

Kapitel 13: Wasserstoffspeicherkraftwerke

\section{Dr.-Ing. Niklas Hartmann}

Dr.-Ing. Niklas Hartmann studierte Wirtschaftsingenieurwesen mit der Fachrichtung Maschinenbau an der TU Kaiserslautern. Von 2008 bis Ende 2012 promovierte Herr Hartmann zum Thema „Rolle und Bedeutung der Stromspeicher bei hohen Anteilen erneuerbarer Energien in Deutschland -Speichersimulation und Betriebsoptimierung " am Institut für Energiewirtschaft und Rationelle Energieanwendung (IER) der Universität Stuttgart. Seit Januar 2013 ist Herr Hartmann wissenschaftlicher Mitarbeiter am Fraunhofer-Institut für Solare Energiesysteme ISE und seit Januar 2015 Teamleiter des Teams „Energiesysteme und -märkte“. Der Schwerpunkt seiner wissenschaftlichen Arbeit liegt in der Systemanalyse von Energiesystemen mit dem Fokus der Integration hoher Anteile erneuerbarer Energien in das Energiesystem Deutschlands.

Internet: www.ise.fraunhofer.de

Kapitel 7: Stromerzeugung aus Windenergie

Kapitel 8: Photovoltaik

\section{Dipl.-Ing. Wilfried Hennings}

Studium der Elektrotechnik an der RWTH Aachen. Seit 1979 wissenschaftlicher Mitarbeiter am Forschungszentrums Jülich, seit 2008 am Institut für Energie- und Klimaforschung - Systemforschung und Technologische Entwicklung (IEK-STE).

Schwerpunkte: Integration von erneuerbaren Energien, Speichern und Elektrofahrzeugen im Stromversorgungssystem.

Internet: www.fz-juelich.de

Kapitel 21: Elektromobilität

\section{M . Sc. Noha Saad Hussein}

Noha Saad Hussein hat einen Bachelor in Architekturingenieurwesen und Umwelttechnik und einen Masterabschluss in erneuerbaren Energien und Energieeffizienz an der Universität Kassel. Sie hat ihre Masterarbeit am Fraunhofer ISE zu dem Thema lokales Wertschöpfungspotenzial von CSP und Windkraftanlagen in Ägypten geschrieben. Seitdem arbeitet sie am Fraunhofer ISE als wissenschaftliche Mitarbeiterin im Team „Energiesysteme und -märkte“. Ihr Hauptfokus liegt in der Energiesystemanalyse von Verteilnetzen 
mit Schwerpunkt auf dem Wärmesektor und den Strom-Wärme Kopplungstechnologien. Zusätzlich arbeitet sie daran, erneuerbare Energietechnologien techno-ökonomisch zu bewerten und das lokale Wertschöpfungspotenzial zu identifizieren. Weiterhin untersucht sie alternative Lösungen erneuerbarer Versorgung der unterschiedlichen Verbrauchssektoren.

Internet: www.ise.fraunhofer.de

Kapitel 7: Stromerzeugung aus Windenergie

Kapitel 8: Photovoltaik

\section{Eng. Verena Jülch}

Studium Energie- und Umweltmanagement an der Universität Flensburg. Seit 2011 ist sie als wissenschaftliche Mitarbeiterin am Fraunhofer-Institut für Solare Energiesysteme ISE in Freiburg, zunächst im Bereich solar betriebene Meerwasserentsalzungsanlagen, dann im Team „Energiesysteme und -märkte“ tätig.

Schwerpunkte: Techno-ökonomische Analyse von Photovoltaik, anderen erneuerbaren Energien und Stromspeichertechnologien in Deutschland und weltweit.

Internet: www.ise.fraunhofer.de

Kapitel 7: Stromerzeugung aus Windenergie

Kapitel 8: Photovoltaik

\section{Dipl.-Ing. Dipl.-Wirtsch.-Ing. Erika Kämpf}

Studium der elektrischen Energietechnik an der Technischen Hochschule Karlsruhe (heute: KIT), wirtschaftswissenschaftliches Zusatzstudium an der RWTH Aachen. Von 2005 bis 2010 Projektingenieurin bei Siemens Energy Automation: Mitwirkung an Entwicklungsprojekten für Verteilnetz-Analyse-Anwendungen (Distribution Management Systems) und dem Smart Grid Projekt E-DeMa, Fabriktests und weltweite Vor-Ort-Inbetriebnahme von Energie-Management-Systemen für Übertragungsnetzbetreiber. Seit 2011 wissenschaftliche Mitarbeit und Projektleitung am Fraunhofer IWES sowie Promotion im Bereich „Blindleistungsbereitstellung von Verteilnetzen an Übertragungsnetze“.

Internet: www.uni-kassel.de, www.iwes.fraunhofer.de

Kapitel 15: Elektrische Verteilungsnetze im Wandel

\section{Dipl.-Ing. Philipp Klever}

Studium der Umwelttechnik und Ressourcenmanagement an der Ruhr-Universität Bochum. Von 2012 bis 2014 wissenschaftlicher Mitarbeiter am Forschungszentrums Jülich, Institut für Energie- und Klimaforschung - Systemforschung und technologische Entwicklung (IEK-STE). Seit April 2015 geschäftsführender Gesellschafter der Firma enerion $\mathrm{GmbH} \& \mathrm{Co} . \mathrm{KG}$ im Bereich Energie- und Ressourceneffizienz für Unternehmen.

Schwerpunkte: wirtschaftliche und technische Bewertung effizienter Energieerzeugungsanlagen im Gebäudesektor.

Internet: www.fz-juelich.de

Kapitel 16: Mikro-Kraftwärmekopplungsanlagen (Mikro-KWK) 


\section{Sc. Markus Kraiczy}

Markus Kraiczy studierte Elektro- und Informationstechnik an der Hochschule Anhalt, elektrische Energietechnik an der Universität von Süddänemark und regenerative Energien und Energieeffizienz an der Universität Kassel. Er ist seit September 2012 als wissenschaftlicher Mitarbeiter am Fraunhofer Institut für Windenergie und Energiesystemtechnik (IWES) in Kassel in der Abteilung „Betrieb Verteilungsnetze“ tätig.

Forschungsschwerpunkte: Parallelbetrieb lokal geregelter Netzbetriebsmittel und dezentraler Erzeugungsanlagen, Maßnahmen der statischen Spannungshaltung und des Blindleistungsmanagements in Netzplanung und Netzbetrieb.

Internet: www.iwes.fraunhofer.de

Kapitel 15: Elektrische Verteilungsnetze im Wandel

\section{Dipl.-Ing. Jochen Linssen}

Studium des Maschinenbaus an der RWTH Aachen, Fachrichtung Kraftfahrwesen. Seit 2000 wissenschaftlicher Mitarbeiter am Forschungszentrums Jülich, Institut für Energie- und Klimaforschung - Systemforschung und technologische Entwicklung (IEKSTE)

Schwerpunkte: Kraftstoffstrategien und Energiespeicher.

Internet: www.fz-juelich.de

Kapitel 10: Elektrochemische Speicher

Kapitel 21: Elektromobilität

\section{Dr. Peter Markewitz}

Der Energie- und Verfahrenstechniker Dr. Peter Markewitz ist Wissenschaftler am Forschungszentrum Jülich. Er leitet die Arbeitsgruppe Energietechnik im Bereich Systemforschung und Technologische Entwicklung des Instituts für Energie- und Klimaforschung. Seine Forschungsfelder sind Energiesystemanalysen, Klimagasreduktionsstrategien, Technologiebewertungen sowie Kraftwerkstechnik.

Internet: www.fz-juelich.de

Kapitel 1: Motivation, Zielsetzung und Technologieauswahl

Kapitel 2: Zukünftige Energiewelt - Szenarien und robuste Trends

Kapitel 3: Kohlekraftwerke

Kapitel 4: Gaskraftwerke

Kapitel 5: $\mathrm{CO}_{2}$-Abscheidung

Kapitel 6: $\mathrm{CO}_{2}$-Nutzung

\section{Dipl.-Kffr. Julia Michaelis}

Studium „Europäische Wirtschaft“ an der Otto-Friedrich-Universität Bamberg und der École de Management Strasbourg, Frankreich mit Schwerpunkt in Statistik. 2011 Studienabschluss mit Diplomarbeit bei der PricewaterhouseCoopers AG. Von Juni bis Dezember 2011 als wissenschaftliche Mitarbeiterin im Competence Center Energiepolitik und Energiesysteme am Fraunhofer Institut für System- und Innovationsforschung ISI, seit 
Januar 2012 im Competence Center Energietechnologien und Energiesysteme. Seit September 2012 Doktorandin am Lehrstuhl für Energiewirtschaft der Technischen Universität Dresden. Forschungstätigkeit im Bereich Energiesystemanalyse und Wirtschaftlichkeitsbewertung von Energiespeichern sowie Produktionsverfahren von Wasserstoff.

Internet: www.isi.fraunhofer.de

Kapitel 12: Power-to-Gas

\section{Dr. Thomas Schlegl}

Studium der Physik an der Universität Regensburg und der Université Montpellier II. Promotion zum Dr. rer. nat. an der Universität Regensburg in Zusammenarbeit mit dem Fraunhofer-Institut für Solare Energiesysteme ISE. Seit 2005 Leitung der Strategieplanung des Fraunhofer ISE und Geschäftsführer der Fraunhofer-Allianz Energie. In 2009 Gründung der Fraunhofer Forschungsgruppe „Energiesystemanalyse“. Schwerpunkt sind Technoökonomische Bewertung von Energietechnologien, Marktanalysen und Geschäftsmodelle, Kraftwerkseinsatzplanung und Betriebsstrategien sowie nationale und regionale Energieversorgungskonzepte.

Internet: www.ise.fraunhofer.de

Kapitel 7: Stromerzeugung aus Windenergie

Kapitel 8: Photovoltaik

\section{Dr.-Ing. Peter Stenzel}

Studium Umwelttechnik und Ressourcenmanagement an der Ruhr-Universität Bochum. Anschließend wissenschaftlicher Mitarbeiter am Zentrum für Innovative Energiesysteme (ZIES) an der Fachhochschule Düsseldorf. Promotion zum Dr.-Ing. an der Ruhr-Universität Bochum am Lehrstuhl für Energiesysteme und Energiewirtschaft (LEE) im Jahr 2011. Seit Ende 2011 wissenschaftlicher Mitarbeiter am Forschungszentrum Jülich, Institut für Energie- und Klimaforschung - Systemforschung und technologische Entwicklung (IEKSTE).

Schwerpunkte: Techno-ökonomischen Analyse und Bewertung von Energiespeichern, Stromerzeugung mit Osmoseverfahren.

Internet: www.fz-juelich.de

Kapitel 10: Elektrochemische Speicher

\section{Dipl.-Ing. Friedrich Schulte (RWE AG, Konzern F\&E, Leiter Technologien/Strategie)}

Friedrich Schulte ist im RWE Konzern verantwortlich für die Strategie in Forschung \& Entwicklung sowie „Technology Foresight“. Im Mittelpunkt seiner Arbeit steht die kontinuierliche Bewertung aller technologischen Entwicklungen mit Relevanz für die Energiewirtschaft sowie die Ableitung und Umsetzung der daraus folgenden F\&E-Bedarfe.

Friedrich Schulte arbeitet seit 1992 in der Energiewirtschaft und hat dabei in unterschiedlichen Management - und Projektrollen Expertise zu zahlreichen Technologien u. a. aus IT, Kommunikationstechnik, Stromnetzen, Stromerzeugung und Energieanwendung 
gewonnen. Seit 2003 liegt sein Arbeitsschwerpunkt auf Innovationen und F\&E wobei er auch als Geschäftsführer einer Venture Capital Gesellschaft für Energietechnologien tätig war. Seine aktuelle Funktion umfasst auch Kooperationen von Partnern aus Industrie und Wissenschaft auf internationaler Ebene der Energieforschung.

Herr Schulte hat seinen Abschluss als Dipl.-Ing. Elektrotechnik 1986 an der TU Dortmund erworben und war dort zunächst als wissenschaftlicher Mitarbeiter an der Fakultät für Nachrichtentechnik tätig. Ab 1988 entwickelte er im Industrieauftrag elektronische Systeme am Fraunhofer Institut in Duisburg.

Internet: www.rwe.com

Kapitel 1: Motivation, Zielsetzung und Technologieauswahl

\section{Dipl. Wi.-Ing. Jan Steinbach}

Jan Steinbach arbeitet seit 2009 als wissenschaftlicher Mitarbeiter am Fraunhofer ISI in der Abteilung Energiepolitik und Energiemärkte. Er hat Wirtschaftsingenieurwesen am Karlsruher Institut für Technologie (KIT) und an der Universiteit Maastricht mit den Schwerpunkten Energie- und Umwelttechnik, Internationale Wirtschaftspolitik und Finanz- und Rechnungswesen studiert.

Forschungsschwerpunkte sind die modelgestützte Bewertung von Politikinstrumenten zur Förderung von erneuerbaren Energien und Energieeffizienz in Gebäuden. Dazu promoviert er am Institut für Industriebetriebslehre und industrielle Produktion (KIT). Von September bis Dezember 2012 war er Gastwissenschaftler am Lawrence Berkeley National Laboratory in Kalifornien (USA).

Internet: www.isi.fraunhofer.de

Kapitel 17: Raumlufttechnik und Klimakältesysteme

\section{Dipl.-Wirtsch.-Ing. (FH) Michael Taumann}

Michael Taumann studierte den Studiengang Sustainable Energy Competence (SENCE) an der Hochschule Rottenburg. Von April 2012 bis Dezember 2012 verfasste Herr Taumann seine Masterarbeit zum Thema „Modellierung des Zubaus erneuerbarer Stromerzeugungstechnologien in Deutschland“ am Fraunhofer-Institut für Solare Energiesysteme ISE. Bis April 2013 war Herr Taumann weiterhin als geprüfte wissenschaftliche Hilfskraft am Fraunhofer ISE tätig. Seit Mai 2013 ist Herr Taumann wissenschaftlicher Mitarbeiter am Zentrum für Sonnenenergie- und Wasserstoff-Forschung Baden-Württemberg. Der Schwerpunkt seiner derzeitigen Arbeit liegt in der Analyse von Biomasse-KWK in BadenWürttemberg und der Erfahrungsberichte gemäß $§ 65$ EEG.

Internet: www.zsw-bw.de

Kapitel 7: Stromerzeugung aus Windenergie

\section{Dr. phil. nat. Sandra Ullrich}

Studium der Biochemie in Leipzig und Frankfurt mit Vertiefung im Bereich Biophysikalische Chemie zur Diplomarbeit im Jahr 2008. Abschluss der Promotion in Biochemie über Funktion und Struktur von Membranproteinen mittels Festkörper-Nuklearmagnetischer 
Resonanz-Spektroskopie (NMR) im Jahr 2012. Anschließend wissenschaftliche Mitarbeiterin an der Goethe Universität und des Biomolekularen Magnetresonanz Zentrums (BMRZ) in Frankfurt. Seit April 2014 wissenschaftliche Mitarbeiterin und Projektleiterin am Competence Center Energietechnologien und Energiesysteme des Fraunhofer-Instituts für System- und Innovationforschung ISI in Karlsruhe. Ihre Forschungsschwerpunkte sind Elektromobilität, Analyse und Modellierung von Energiesystemen sowie soziale Implikationen und Verteilungseffekte der Energiewende im nationalen und europäischen Kontext.

Internet: www.isi.fraunhofer.de

Kapitel 1: Motivation, Zielsetzung und Technologieauswahl

Kapitel 14: Übertragungsnetze

\section{Prof. Dr. Martin Wietschel}

Studium des Wirtschaftsingenieurwesens an der Universität Karlsruhe. Promotion und Habilitation mit dem Abschluss der venia legendi für Betriebswirtschaftslehre an derselben Universität am Institut für Industriebetriebslehre und Industrielle Produktion. Von 2002 bis 2011 wissenschaftlicher Mitarbeiter und Projektleiter am Fraunhofer-Institut für System- und Innovationsforschung ISI im Competence Center Energiepolitik und Energiesysteme und seit 2007 Leiter des Geschäftsfelds Energiewirtschaft. Seit Januar 2012 stellvertretender Leiter des Competence Centers Energietechnologien und Energiesysteme sowie Leiter des Geschäftsfelds Energiewirtschaft. Von 2005 bis 2011 hatte er einen Lehrauftrag an der ETH Zürich und im Jahre 2008 wurde ihm eine außerplanmäßige Professur an der Universität Karlsruhe (heutiges Karlsruher Institut für Technologie (KIT)) verliehen.

Seine Forschungsschwerpunkte sind die Nachhaltige Energiewirtschaft, die Bewertung neuer Energietechnologien, Energiesystemanalysen sowie innovative Kraftstoffe und Antriebe im Verkehr. Weiterhin ist er in verschiedenen Gremien zur Elektromobilität, wie der Nationalen Plattform Elektromobilität, vertreten.

Internet: www.isi.fraunhofer.de

Kapitel 1: Motivation, Zielsetzung und Technologieauswahl 


\section{Inhaltsverzeichnis}

\section{Teil I Einleitende Betrachtungen}

1 Motivation, Zielsetzung und Technologieauswahl . . . . . . . . . . 3

Sandra Ullrich, Martin Wietschel, Tobias Bischkowski, Friedrich Schulte und Peter Markewitz

1.1 Abkürzungen . . . . . . . . . . . . . . . . . . . 11

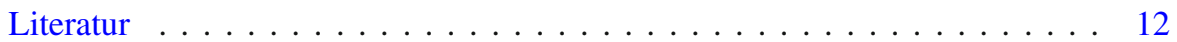

$2 \quad$ Zukünftige Energiewelt - Szenarien und robuste Trends . . . . . . . . . . 13

Klaus Biß, Peter Markewitz und Ulf Birnbaum

2.1 Globale Energiebedarfsentwicklung . . . . . . . . . . . . . . . 13

2.2 EU-27-Energieszenarien . . . . . . . . . . . . . . . . 21

2.3 Nationale Energieszenarien . . . . . . . . . . . . . . . 25

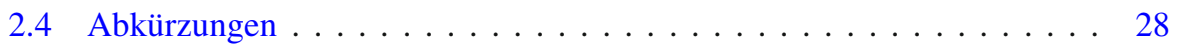

Literatur . . . . . . . . . . . . . . . . . . . . . . . . . . . . 29

\section{Teil II Kraftwerkstechnik für fossile Brennstoffe plus CCS-Abscheidetechnik}

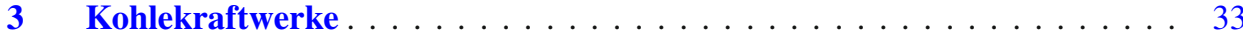

Peter Markewitz, Richard Bongartz und Klaus Biß

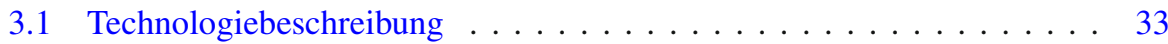

3.1 .1 Funktionale Beschreibung . . . . . . . . . . . . . . 35

3.1 .2 Status quo und Entwicklungsziele . . . . . . . . . . . . . 38

3.1.3 Technische Kenndaten . . . . . . . . . . . . . . . . . . . 38

3.2 Zukünftige Anforderungen und Randbedingungen . . . . . . . . . . . . . . . . . . . . . . . . . . . . . . .

3.2 .1 Gesellschaft . . . . . . . . . . . . . . . . . 41

3.2 .2 Kostenentwicklung . . . . . . . . . . . . . . . . . . . 42

3.2 .3 Politik und Regulierung . . . . . . . . . . . . . . . . . . . . . . . . . . . . . . . . . . . . 43

3.2 .4 Marktrelevanz . . . . . . . . . . . . . . . . . . . . . . 44

3.2.5 Mögliche Wechselwirkungen mit anderen Technologien . . . . . . 45

3.2 .6 Game Changer . . . . . . . . . . . . . . . . . . . . . . . 45 
3.3 Technologieentwicklung . . . . . . . . . . . . . . . . . 46

3.3.1 Entwicklungsziele . . . . . . . . . . . . . . . . 46

3.3.2 F\&E-Bedarf und kritische Entwicklungshemmnisse . . . . . . . . . . 46

3.4 Abkürzungen . . . . . . . . . . . . . . . . . . 54

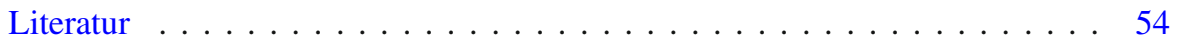

4 Gaskraftwerke . . . . . . . . . . . . . . . . . . . 57

Peter Markewitz, Richard Bongartz und Klaus Biß

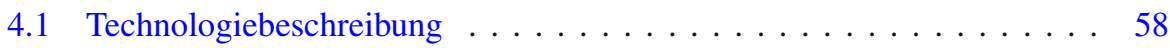

4.1 .1 Funktionale Beschreibung . . . . . . . . . . . . . . . 58

4.1.2 Status quo und Entwicklungsziele . . . . . . . . . . . . . . 61

4.1 .3 Technische Kenndaten . . . . . . . . . . . . . . . . . . 62

4.2 Zukünftige Anforderungen und Randbedingungen . . . . . . . . . . . . . . 64

4.2 .1 Gesellschaft . . . . . . . . . . . . . . . . . . . . . 64

4.2 .2 Kostenentwicklung . . . . . . . . . . . . . . . . . . . . . 64

4.2 .3 Politik und Regulierung . . . . . . . . . . . . . . . . . . . . . . . . . . . . . 65

4.2 .4 Marktrelevanz . . . . . . . . . . . . . . . . . . . 65

4.2.5 Mögliche Wechselwirkungen mit anderen Technologien . . . . . . 66

4.2 .6 Game Changer . . . . . . . . . . . . . . . . . . . . . 67

4.3 Technologieentwicklung . . . . . . . . . . . . . . . . 67

4.3.1 Entwicklungsziele ................. 67

4.3.2 F\&E-Bedarf und kritische Entwicklungshemmnisse . . . . . . . 68

4.4 Abkürzungen . . . . . . . . . . . . . . . . . . . . . . 74

Literatur . . . . . . . . . . . . . . . . . . . . . . . 74

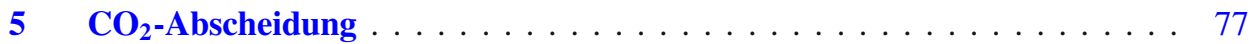

Richard Bongartz, Peter Markewitz und Klaus Biß

5.1 Technologiebeschreibung . . . . . . . . . . . . . . . . . . 77

5.1 .1 Funktionale Beschreibung . . . . . . . . . . . . . . . . 77

5.1 .2 Status quo und Entwicklungsziele . . . . . . . . . . . . . . . . . . . . . . . . . . . . . . . .

5.1 .3 Technische Kenndaten . . . . . . . . . . . . . . . . 81

5.2 Zukünftige Anforderungen und Randbedingungen . . . . . . . . . . . . . . 82

5.2 .1 Gesellschaft . . . . . . . . . . . . . . . . . . . 82

5.2 .2 Kostenentwicklung . . . . . . . . . . . . . . . . . . . . . . . 82

5.2 .3 Politik und Regulierung . . . . . . . . . . . . . . . . . . 83

5.2 .4 Marktrelevanz . . . . . . . . . . . . . . . . . . . . 84

5.2.5 Mögliche Wechselwirkungen mit anderen Technologien . . . . . . 84

5.2 .6 Game Changer . . . . . . . . . . . . . . . . . . . . 84

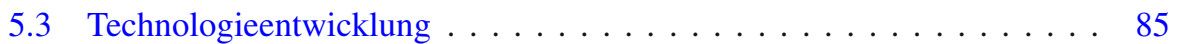

5.3 .1 Entwicklungsziele . . . . . . . . . . . . . . . 85

5.3.2 F\&E-Bedarf und kritische Entwicklungshemmnisse . . . . . . 85 
5.4 Abkürzungen . . . . . . . . . . . . . . . . . . . . . . 90

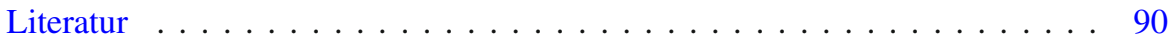

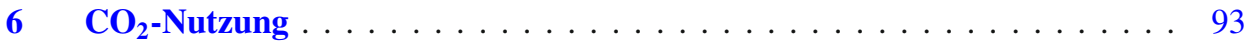

Richard Bongartz, Peter Markewitz und Klaus Biß

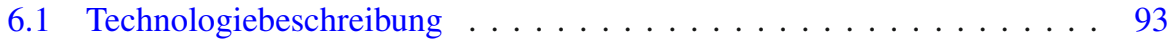

6.1.1 Funktionale Beschreibung . . . . . . . . . . . . . . . 93

6.1 .2 Status quo und Entwicklungsziele . . . . . . . . . . . . . . . . . . . . . . 95

6.1 .3 Technische Kenndaten . . . . . . . . . . . . . . . . . . 96

6.2 Zukünftige Anforderungen und Randbedingungen . . . . . . . . . . . . . . . . . . . . . . . . . . . . . 97

6.2 .1 Gesellschaft . . . . . . . . . . . . . . . . . . 97

6.2 .2 Kostenentwicklung . . . . . . . . . . . . . . . . . . . 97

6.2 .3 Politik und Regulierung . . . . . . . . . . . . . . . . . . . . . . 98

6.2 .4 Marktrelevanz . . . . . . . . . . . . . . . . . . . . . 98

6.2.5 Mögliche Wechselwirkungen mit anderen Technologien . . . . . . 98

6.2 .6 Game Changer . . . . . . . . . . . . . . . . . . . . . . . . 98

6.3 Technologieentwicklung . . . . . . . . . . . . . . . . . . . . . . 99

6.3 .1 Entwicklungsziele . . . . . . . . . . . . . . . . . . 99

6.3.2 F\&E-Bedarf und kritische Entwicklungshemmnisse . . . . . . . . 99

6.4 Abkürzungen . . . . . . . . . . . . . . . . . . . . . . . . . . 99

Literatur . . . . . . . . . . . . . . . . . . . . . . 100

\section{Teil III Erneuerbare Energietechnologien}

7 Stromerzeugung aus Windenergie . . . . . . . . . . . . . . . . . 103

Niklas Hartmann, Noha Saad Hussein, Michael Taumann, Verena Jülch und Thomas Schlegl

7.1 Technologiebeschreibung . . . . . . . . . . . . . . . . 103

7.1.1 Funktionale Beschreibung . . . . . . . . . . . . . . 105

7.1.2 Status quo und Entwicklungsziele . . . . . . . . . . . . . . 108

7.1.3 Technische Kenndaten . . . . . . . . . . . . . . . . . . . . . . 110

7.2 Zukünftige Anforderungen und Randbedingungen . . . . . . . . . . . . . 111

7.2 .1 Gesellschaft . . . . . . . . . . . . . . . . . 111

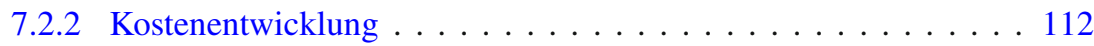

7.2 .3 Politik und Regulierung . . . . . . . . . . . . . . . . . . . . 113

7.2 .4 Marktrelevanz . . . . . . . . . . . . . . . . . . . 113

7.2.5 Mögliche Wechselwirkungen mit anderen Technologien . . . . . . 114

7.2 .6 Game Changer . . . . . . . . . . . . . . . . . 115

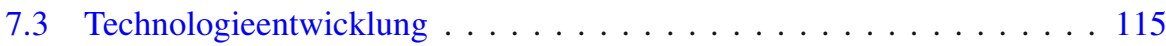

7.3.1 Entwicklungsziele . . . . . . . . . . . . . . . 115

7.3.2 F\&E-Bedarf und kritische Entwicklungshemmnisse . . . . . . 116 
7.4 Abkürzungen . . . . . . . . . . . . . . . . . . . . . . . . 120

Literatur . . . . . . . . . . . . . . . . . . . . . . . . . . 120

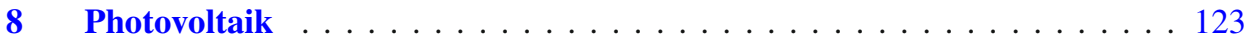

Verena Jülch, Niklas Hartmann, Noha Saad Hussein und Thomas Schlegl

8.1 Technologiebeschreibung . . . . . . . . . . . . . . 123

8.1 .1 Funktionale Beschreibung . . . . . . . . . . . . . 123

8.1.2 Status quo und Entwicklungsziele . . . . . . . . . . . . . . 125

8.1.3 Technische Kenndaten . . . . . . . . . . . . . . . . . . . . . 127

8.2 Zukünftige Anforderungen und Randbedingungen . . . . . . . . . . . . . 128

8.2 .1 Gesellschaft . . . . . . . . . . . . . . . . . . . . . . 128

8.2 .2 Kostenentwicklung . . . . . . . . . . . . . . . . . . . . . . . . . . . . . . . . . . . . . . . . . . . . . . .

8.2 .3 Politik und Regulierung . . . . . . . . . . . . . . . . . . . . . 130

8.2 .4 Marktrelevanz . . . . . . . . . . . . . . . . . . 130

8.2.5 Mögliche Wechselwirkungen mit anderen Technologien . . . . . . 131

8.2 .6 Game Changer . . . . . . . . . . . . . . . . . . . . 131

8.3 Technologieentwicklung . . . . . . . . . . . . . . . . . 132

8.3.1 Entwicklungsziele . . . . . . . . . . . . . . . 132

8.3.2 F\&E-Bedarf und kritische Entwicklungshemmnisse . . . . . . . 133

8.4 Abkürzungen . . . . . . . . . . . . . . . . . . . . . . . . . 137

Literatur . . . . . . . . . . . . . . . . . . . . . 137

9 Solarthermische Kraftwerke . . . . . . . . . . . . . . . . . . . . . . . . . . . 139

Reiner Buck

9.1 Technologiebeschreibung . . . . . . . . . . . . . . . . . . 139

9.1.1 Funktionale Beschreibung . . . . . . . . . . . . . . . . . . . . 139

9.1.2 Status quo und Entwicklungsziele . . . . . . . . . . . . . . . . . 142

9.1.3 Technische Kenndaten . . . . . . . . . . . . . . . . . . . . . . 145

9.2 Zukünftige Anforderungen und Randbedingungen . . . . . . . . . . . . . 147

9.2.1 Gesellschaft . . . . . . . . . . . . . . . . . . . . . . . 147

9.2 .2 Kostenentwicklung . . . . . . . . . . . . . . . . . . . . 147

9.2.3 Politik und Regulierung . . . . . . . . . . . . . . . . . . . . . . . . 148

9.2 .4 Marktrelevanz . . . . . . . . . . . . . . . . . . . . . . . . . . . 149

9.2.5 Mögliche Wechselwirkungen mit anderen Technologien . . . . . . 149

9.2 .6 Game Changer . . . . . . . . . . . . . . . . . . . . . . . . . . 150

9.3 Technologieentwicklung . . . . . . . . . . . . . . . . . . 150

9.3.1 Entwicklungsziele . . . . . . . . . . . . . . . . . . 150

9.3.2 F\&E-Bedarf und kritische Entwicklungshemmnisse . . . . . . . 151

9.4 Abkürzungen . . . . . . . . . . . . . . . . . . . . . . . . . . . . . 154

Literatur . . . . . . . . . . . . . . . . . . . . . . . . . . . . 154 


\section{Teil IV Energiespeicher}

10 Elektrochemische Speicher . . . . . . . . . . . . . . . . . . . . . . 157

Peter Stenzel, Johannes Fleer und Jochen Linssen

10.1 Technologiebeschreibung . . . . . . . . . . . . . . . . 157

10.1.1 Funktionale Beschreibung . . . . . . . . . . . . . . . . 157

10.1.2 Status quo und Entwicklungsziele . . . . . . . . . . . . . . 168

10.1.3 Technische Kenndaten . . . . . . . . . . . . . . . . . . . . . 174

10.2 Zukünftige Anforderungen und Randbedingungen . . . . . . . . . . . . . 181

10.2.1 Gesellschaft . . . . . . . . . . . . . . . . . 181

10.2.2 Kostenentwicklung . . . . . . . . . . . . . . . . . . . . 182

10.2.3 Politik und Regulierung . . . . . . . . . . . . . . . . . . . 187

10.2.4 Marktrelevanz . . . . . . . . . . . . . . . . . . . . . . . . . . 191

10.2.5 Mögliche Wechselwirkungen mit anderen Technologien . . . . . . 197

10.2.6 Game Changer . . . . . . . . . . . . . . . . . . . . . . . . . 198

10.3 Technologieentwicklung . . . . . . . . . . . . . . . . . . . . . . . 199

10.3.1 Entwicklungsziele . . . . . . . . . . . . . . . . . . . . . 199

10.3.2 F\&E-Bedarf und kritische Entwicklungshemmnisse . . . . . . . . 200

10.4 Abkürzungen . . . . . . . . . . . . . . . . . . . . . . . . . . 209

Literatur . . . . . . . . . . . . . . . . . . . . . . . . . . . . . . . . . . . 209

11 Druckluftspeicher . . . . . . . . . . . . . . . . . 215

Fabio Genoese

11.1 Technologiebeschreibung . . . . . . . . . . . . . . . 215

11.1.1 Funktionale Beschreibung . . . . . . . . . . . . . . . . 215

11.1.2 Status quo und Entwicklungsziele . . . . . . . . . . . . . . . . . 218

11.1.3 Technische Kenndaten . . . . . . . . . . . . . . . . . . . . . . . . . . 219

11.2 Zukünftige Anforderungen und Randbedingungen . . . . . . . . . . . . 220

11.2.1 Gesellschaft . . . . . . . . . . . . . . . . . . . 220

11.2.2 Kostenentwicklung . . . . . . . . . . . . . . . . . . . . 220

11.2.3 Politik und Regulierung . . . . . . . . . . . . . . . . . . . . . . . . . . . . . . . . . . . . . . . . . . . . . . .

11.2.4 Marktrelevanz . . . . . . . . . . . . . . . . . . . . . . 222

11.2.5 Mögliche Wechselwirkungen mit anderen Technologien . . . . . . 223

11.3 Game Changer . . . . . . . . . . . . . . . . . . . . . 223

11.4 Technologieentwicklung . . . . . . . . . . . . . . . . . . . 223

11.4.1 Entwicklungsziele ..................... 223 
11.5 F\&E-Bedarf und kritische Entwicklungshemmnisse . . . . . . . . . . . . . . . . . . . . . . . . . . . . . . . . . . . . 24

11.6 Abkürzungen . . . . . . . . . . . . . . . . . . . . . . . . . . . 227

Literatur . . . . . . . . . . . . . . . . . . . . . . . . 227

12 Power-to-Gas . . . . . . . . . . . . . . . . . . . . . . . . . . . . . . . 229

Julia Michaelis und Fabio Genoese

12.1 Technologiebeschreibung . . . . . . . . . . . . . . . . . . . . . 229

12.1.1 Funktionale Beschreibung . . . . . . . . . . . . . . . . . . . 229

12.1.2 Status quo und Entwicklungsziele . . . . . . . . . . . . . . . . . . . . . . . . . . . . . . . . . . . .

12.1.3 Technische Kenndaten . . . . . . . . . . . . . . . . . . 233

12.2 Zukünftige Anforderungen und Randbedingungen . . . . . . . . . . . . . . 234

12.2.1 Gesellschaft . . . . . . . . . . . . . . . . . . . . . . . . . . . . . . . . . . .

12.2.2 Kostenentwicklung . . . . . . . . . . . . . . . . . . . . . . . . . . . . . . . . . . . . . . . . . .

12.2.3 Politik und Regulierung . . . . . . . . . . . . . . . . . . . . . . . . . . . . . . . . . . . . . . . . . . . . . . . .

12.2.4 Marktrelevanz . . . . . . . . . . . . . . . . . . . . . . 236

12.2.5 Mögliche Wechselwirkungen mit anderen Technologien . . . . . . 239

12.2.6 Game Changer . . . . . . . . . . . . . . . . . . . . . . . 239

12.3 Technologieentwicklung . . . . . . . . . . . . . . . . . . . . . . 240

12.3.1 Entwicklungsziele . . . . . . . . . . . . . . . . . . 240

12.3.2 F\&E Bedarf und kritische Entwicklungshemmnisse . . . . . . 240

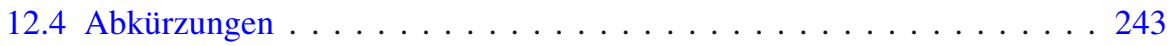

Literatur . . . . . . . . . . . . . . . . . . . . . . . . 243

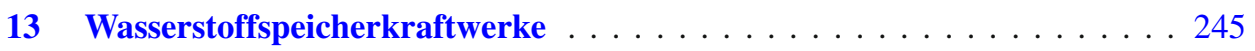

Fabio Genoese

13.1 Technologiebeschreibung . . . . . . . . . . . . . . . . . 245

13.1.1 Funktionale Beschreibung . . . . . . . . . . . . . . . . . . . . . . . . . . . . . . . . . . . . .

13.1.2 Status quo und Entwicklungsziele . . . . . . . . . . . . . . . . . 249

13.1.3 Technische Kenndaten . . . . . . . . . . . . . . . . . 251

13.2 Zukünftige Anforderungen und Randbedingungen . . . . . . . . . . . . 254

13.2.1 Gesellschaft . . . . . . . . . . . . . . . . . . . . . . . . . . . . . . . . . . . . . . .

13.2.2 Kostenentwicklung . . . . . . . . . . . . . . . . . . . . . . . . . . . . . . . . . . . . . . . . . . .

13.2.3 Politik und Regulierung . . . . . . . . . . . . . . . . . . . . . . . . . . . . . . . . . . . . . . . . . . . .

13.2.4 Marktrelevanz . . . . . . . . . . . . . . . . . . . 256

13.2.5 Mögliche Wechselwirkungen mit anderen Technologien . . . . . . 257

13.2.6 Game Changer . . . . . . . . . . . . . . . . . . . . . . . . . . . . . . . . . . . . . . . . . . . . . . .

13.3 Technologieentwicklung . . . . . . . . . . . . . . . . . . . 258

13.3.1 Entwicklungsziele . . . . . . . . . . . . . . 258

13.3.2 F\&E-Bedarf und kritische Entwicklungshemmnisse . . . . . . . 258

13.4 Abkürzungen . . . . . . . . . . . . . . . . . . . . . 262

Literatur . . . . . . . . . . . . . . . . . . . . . 262 


\section{Teil V Elektrizitätsnetze}

14 Übertragungsnetze . . . . . . . . . . . . . . . . . . . . . . . 267

Sandra Ullrich

14.1 Dynamisierung der Übertragungskapazität . . . . . . . . . . . . . . . . . . . . . . . . . . . . . . 267

14.1.1 Technologiebeschreibung . . . . . . . . . . . . . . . 267

14.1.2 Zukünftige Anforderungen und Randbedingungen . . . . . . . . . . 274

14.1.3 Technologieentwicklung . . . . . . . . . . . . . . . 278

14.2 Flexible Drehstromübertragungstechnik . . . . . . . . . . . . . . . . . . . . . . . . . 283

14.2.1 Technologiebeschreibung . . . . . . . . . . . . . . . 283

14.2.2 Zukünftige Anforderungen und Randbedingungen . . . . . . . . . . 290

14.2.3 Technologieentwicklung . . . . . . . . . . . . . . . . . . . . . . 294

14.3 Hybride AC/DC-Netzstrukturen . . . . . . . . . . . . . . . . . . . . . . 298

14.3.1 Technologiebeschreibung . . . . . . . . . . . . . . . . . . 298

14.3.2 Zukünftige Anforderungen und Randbedingungen . . . . . . . . . . 303

14.3.3 Technologieentwicklung . . . . . . . . . . . . . . . . . . . 308

14.4 Exkurs: Supraleiter . . . . . . . . . . . . . . . . . . . . . . 311

14.4.1 HTSL-Strombegrenzer . . . . . . . . . . . . . . . . . . . . . . . . . . . . . . . . . . . . . . . . . . . . . .

14.4.2 HTSL-Kabel . . . . . . . . . . . . . . . . . . . . . 312

14.4.3 Politik und Regulierung . . . . . . . . . . . . . . . . . . . . . . . . . . . . . . . . . . . . . . . . .

14.4.4 Marktrelevanz . . . . . . . . . . . . . . . . . . . . 313

14.4.5 F\&E-Bedarf und kritische Entwicklungshemmnisse . . . . . . . 314

14.5 Abkürzungen . . . . . . . . . . . . . . . . . . . . . . . . . . . . . . . . . . 315

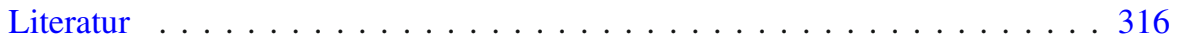

15 Elektrische Verteilungsnetze im Wandel . . . . . . . . . . . . . . . . . 323

Martin Braun, Erika Kämpf und Markus Kraiczy

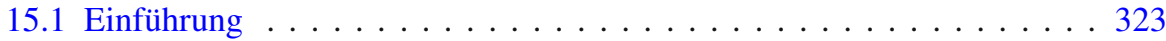

15.2 Bereitstellung von Systemdienstleistungen und IKT-Infrastruktur . . . . . 328

15.3 Netzbetriebsmittel und beeinflussbare Kundenanlagen . . . . . . . . . . . . 331

15.4 Strategische Netzplanung . . . . . . . . . . . . . . . . . . . . . . 334

15.5 Zusammenfassung . . . . . . . . . . . . . . . . . . . . . . . . . . . . . . . . . . . . . . . . . . . . . . . .

15.6 Abkürzungen . . . . . . . . . . . . . . . . . 338

Literatur . . . . . . . . . . . . . . . . . . . . . . . . 339

\section{Teil VI Effizienztechnologien und Mikro-KWK}

16 Mikro-Kraftwärmekopplungsanlagen (Mikro-KWK) . . . . . . . . . . . . . . 347

Ulf Birnbaum, Richard Bongartz und Philipp Klever

16.1 Technologiebeschreibung . . . . . . . . . . . . . . . . . . . 347

16.1.1 Funktionale Beschreibung . . . . . . . . . . . . . . . . . . . . 348

16.1.2 Status quo und Entwicklungsziele . . . . . . . . . . . . . . . 352 
16.1.3 Technische Kenndaten . . . . . . . . . . . . . . . . . . . . . . 354

16.2 Zukünftige Anforderungen und Randbedingungen . . . . . . . . . . . . . 356

16.2.1 Gesellschaft . . . . . . . . . . . . . . . . . . . . . . . . . . . . . . . . . . . . .

16.2.2 Kostenentwicklung . . . . . . . . . . . . . . . . . 356

16.2.3 Politik und Regulierung . . . . . . . . . . . . . . . . . . . . . . . . . . . . . . . . . . . . . . . . . . .

16.2.4 Marktrelevanz . . . . . . . . . . . . . . . . . . . . . 358

16.2.5 Mögliche Wechselwirkungen mit anderen Technologien . . . . . . 359

16.2.6 Game Changer . . . . . . . . . . . . . . . . . . . . . . . . . . 360

16.3 Technologieentwicklung . . . . . . . . . . . . . . 361

16.3.1 Entwicklungsziele . . . . . . . . . . . . . . . . 361

16.3.2 F\&E-Bedarf und kritische Entwicklungshemmnisse . . . . . . . 362

16.4 Abkürzungen . . . . . . . . . . . . . . . . . . . 366

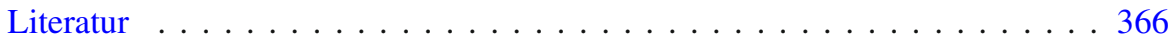

17 Raumlufttechnik und Klimakältesysteme . . . . . . . . . . . . . . . . . . 369

Ali Aydemir und Jan Steinbach

17.1 Technologiebeschreibung . . . . . . . . . . . . . . . . . . . . . . 369

17.1.1 Funktionale Beschreibung . . . . . . . . . . . . . . . . . . 370

17.1.2 Status quo und Entwicklungsziele . . . . . . . . . . . . . . . . 372

17.1.3 Technische Kenndaten . . . . . . . . . . . . . . . . . . . . . . 373

17.2 Zukünftige Anforderungen und Randbedingungen . . . . . . . . . . . . 373

17.2.1 Gesellschaft . . . . . . . . . . . . . . . . . . . 373

17.2.2 Kostenentwicklung . . . . . . . . . . . . . . . . . . . . . . . . . . . 373

17.2.3 Politik und Regulierung . . . . . . . . . . . . . . . . . . . 374

17.2.4 Marktrelevanz . . . . . . . . . . . . . . . . . . . . . . . . 375

17.2.5 Mögliche Wechselwirkungen mit anderen Technologien und

Technologiefeldern . . . . . . . . . . . . . . . . 377

17.2.6 Game Changer . . . . . . . . . . . . . . . . . . . . . . 377

17.3 Technologieentwicklung . . . . . . . . . . . . . . . . . . . 378

17.3.1 Entwicklungsziele . . . . . . . . . . . . . . . 378

17.3.2 F\&E-Bedarf und kritische Entwicklungshemmnisse . . . . . . . 378

17.4 Abkürzungen . . . . . . . . . . . . . . . . . . . 381

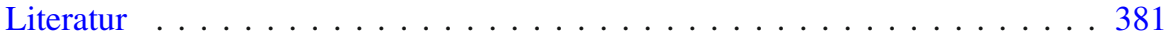

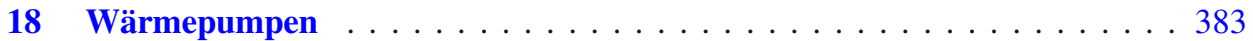

Ali Aydemir

18.1 Technologiebeschreibung . . . . . . . . . . . . . . . . 383

18.1.1 Funktionale Beschreibung . . . . . . . . . . . . . . . . . . . . . . . . . . . . . . 384

18.1.2 Status quo und Entwicklungsziele . . . . . . . . . . . . . . . 385

18.1.3 Technische Kenndaten . . . . . . . . . . . . . . . . . . . . . . 387

18.2 Zukünftige Anforderungen und Randbedingungen . . . . . . . . . . . . 389

18.2.1 Gesellschaft . . . . . . . . . . . . . . . . . . . . . . . . . 389 
18.2.2 Kostenentwicklung . . . . . . . . . . . . . . . . . . . . . . . . 389

18.2.3 Politik und Regulierung . . . . . . . . . . . . . . . . . . . . . 390

18.2.4 Marktrelevanz . . . . . . . . . . . . . . . . . . . . . . . . . . 390

18.2.5 Mögliche Wechselwirkungen mit anderen Technologien . . . . . . 391

18.2.6 Game Changer . . . . . . . . . . . . . . . . . . . . . . . . . 391

18.3 Technologieentwicklung . . . . . . . . . . . . . . . . . . . 392

18.3.1 Entwicklungsziele . . . . . . . . . . . . . . . . . . . 392

18.3.2 F\&E-Bedarf und kritische Entwicklungshemmnisse . . . . . . . 392

18.4 Abkürzungen . . . . . . . . . . . . . . . . . . . . . . . 396

Literatur . . . . . . . . . . . . . . . . . . . . . 396

19 Stromeffizienz in den Sektoren Industrie, GHD und Haushalte . . . . . . 399

Tobias Fleiter

19.1 Technologiebeschreibung . . . . . . . . . . . . . . . . . . . 399

19.1.1 Funktionale Beschreibung . . . . . . . . . . . . . . . . . . . . . . . . 399

19.1.2 Status quo und Entwicklungsziele . . . . . . . . . . . . . . . . . . . . . . . . . . 400

19.2 Zukünftige Anforderungen und Randbedingungen . . . . . . . . . . . . . 405

19.2.1 Gesellschaft . . . . . . . . . . . . . . . . . 405

19.2.2 Kostenentwicklung . . . . . . . . . . . . . . . . . 405

19.2.3 Politik und Regulierung . . . . . . . . . . . . . . . . . . . . 406

19.2.4 Marktrelevanz . . . . . . . . . . . . . . . . . . . . . . 407

19.2.5 Mögliche Wechselwirkungen mit anderen Technologien . . . . . . 407

19.2.6 Game Changer . . . . . . . . . . . . . . . . . . . . . . . . . 408

19.3 Technologieentwicklung . . . . . . . . . . . . . . . . . . . . . . 408

19.3.1 Entwicklungsziele . . . . . . . . . . . . . . . . . . 408

19.3.2 F\&E-Bedarf und kritische Entwicklungshemmnisse . . . . . . . . 409

19.4 Abkürzungen . . . . . . . . . . . . . . . . . . . . 415

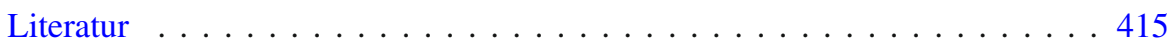

20 Verbrauchssteuerung . . . . . . . . . . . . . . . . . . . . . 417

Nele Friedrichsen

20.1 Technologiebeschreibung . . . . . . . . . . . . . . . . . . . . 417

20.1.1 Funktionale Beschreibung . . . . . . . . . . . . . . . . . . 420

20.1.2 Status quo und Entwicklungsziele . . . . . . . . . . . . . . . . . . . . . . . . . . . . . . . . . . . .

20.1.3 Technische Kenndaten . . . . . . . . . . . . . . . . . . . . . 431

20.2 Zukünftige Anforderungen und Randbedingungen . . . . . . . . . . . . . . . . . . . . . . 431

20.2.1 Gesellschaft . . . . . . . . . . . . . . . . . . 4431

20.2.2 Kostenentwicklung . . . . . . . . . . . . . . . . . . . . . . . . . . . . . . . . . . . . . .

20.2.3 Politik und Regulierung . . . . . . . . . . . . . . . . . . . . . . 435

20.2.4 Marktrelevanz . . . . . . . . . . . . . . . . . . . . . 436

20.2.5 Mögliche Wechselwirkungen mit anderen Technologien . . . . . . 437

20.2.6 Game Changer . . . . . . . . . . . . . . . . . . . . . 437 
20.3 Technologieentwicklung . . . . . . . . . . . . . . . . . . . . . . . . . . . 438

20.3.1 Entwicklungsziele . . . . . . . . . . . . . . . . 438

20.3.2 F\&E-Bedarf und kritische Entwicklungshemmnisse . . . . . . . . . . . . . . . . . . . . . . . . . . . . . . . .

20.4 Abkürzungen . . . . . . . . . . . . . . . . . . . . 442

Literatur . . . . . . . . . . . . . . . . . . . . . . . . . 442

\section{Teil VII Elektromobilität}

21 Elektromobilität . . . . . . . . . . . . . . . . . . . . . . . . . . . . . 447

Wilfried Hennings und Jochen Linssen

21.1 Technologiebeschreibung . . . . . . . . . . . . . . . . . . . . . . . . . 447

21.1.1 Funktionale Beschreibung . . . . . . . . . . . . . . . . . . . . 447

21.1.2 Status quo und Entwicklungsziele . . . . . . . . . . . . . . . . 454

21.1.3 Trendentwicklung der Elektrofahrzeuge . . . . . . . . . . . . . 455

21.2 Zukünftige Anforderungen und Randbedingungen . . . . . . . . . . . . . . . . . . . . . . . . . . . . . . . . . . . .

21.2.1 Gesellschaft . . . . . . . . . . . . . . . . . . . 457

21.2.2 Kostenentwicklung von Elektrofahrzeugen . . . . . . . . . . . 458

21.2.3 Politik und Regulierung . . . . . . . . . . . . . . . . . . . . . . 459

21.2.4 Marktrelevanz . . . . . . . . . . . . . . . . . . . . . . . 461

21.2.5 Mögliche Wechselwirkungen mit anderen Technologien . . . . . . 464

21.2.6 Game Changer . . . . . . . . . . . . . . . . . . . . . . . . . . . . . . . . . . . . . . . . . . .

21.3 Technologieentwicklung . . . . . . . . . . . . . . . . . . . 465

21.3.1 Entwicklungsziele . . . . . . . . . . . . . . . . . 465

21.3.2 F\&E-Bedarf und kritische Entwicklungshemmnisse . . . . . . . 466

21.4 Abkürzungen . . . . . . . . . . . . . . . . . . 470

Literatur ............................. 471

Sachverzeichnis . . . . . . . . . . . . . . . . . . . . 475 